Joint Irish Section and American Society for Nutrition Meeting, 15-17 June 2011, 70th anniversary: 'Vitamins in early development and healthy ageing: impact on infectious and chronic disease'

\title{
101+ Square meals: evaluation
}

\author{
E. Hughes and M. Hennessy \\ Safefood, 7 Eastgate, Avenue Eastgate, Little island, Co. Cork, Republic of Ireland
}

Food poverty is a multidimensional experience, referring to the lack of a nutritionally adequate diet and the related effects on health, culture and social participation ${ }^{(1)}$.

$101+$ Square meals, a recipe book based on healthy eating and budget, was published by the Money, Advice and Budgeting Service (MABS) and Health Service Executive (HSE) in 1998. The recipe book is a well-recognised resource in the Republic of Ireland and it is widely used by Community Dietitians and MABS personnel. The aim of the cookery book is to provide recipes that are: easy-to-prepare, low-budget, yet highly nutritious. The resource also contains useful tips on shopping, home freezing and food safety. In 2008, safefood funded a re-print of $101+$ Square Meals and extended its dissemination to Family Resources Centres (FRC) and Citizens Information Services (CIS). The aim of this evaluation was to certify that the target audience benefit from a cookery book aimed at providing low budget yet nutritious recipes and to ensure that the resource meets all the needs of its user (individuals, families and those delivering home management educational programmes in the community). These findings would then inform the possible development of a resource that would be suitable for the Island of Ireland.

Community dietitians ( $n$ 5) and FRC ( $n$ 2) were invited to participate in the initial stages of this evaluation, which involved a semistructured telephone interview. The aim was to gather their thoughts and opinions on the resource in order to inform a wider quantitative survey. A questionnaire consisting of twelve questions; multiple choice questions and one open-ended question was then developed based on the results of the telephone interviews and was distributed to FRC ( $n$ 38), CIS ( $n$ 15), MAB ( $n$ 60) and community dietitian managers (n 12).

There were eighty-two responses to the questionnaire. All respondents rated the recipe book very highly and considered it an invaluable resource especially in the current recessionary climate. The respondents described the resource being used with a wide range of people, primarily low-income families/people and lone parents, among others and participants would like to see more copies available. The recipes within the resource were rated highly. All of the 82 respondents agreed that the recipes were easy to follow and the ingredients were easy to access. 96\% ( $n$ 79) agreed the recipes were inexpensive to make and $94 \%(n 77)$ agreed the recipes were healthy. The table outlines participant suggestions for improvement of the recipe book.

Respondents recommendations for improvement

Reduce the salt and increase vegetable content of the recipes Improve and update the photographs, illustrations and layout Include more information on budgeting and meal planning.

1. Dowler E (1997) Nutr Soc 56, 51-139. 